\title{
The detection of intramammary infections using online somatic cell counts
}

\author{
Gunnar Dalen, ${ }^{1,2 *}$ Amira Rachah, ${ }^{1}$ Håvard Nørstebø, ${ }^{1,2}$ Ynte H. Schukken, ${ }^{3,4,5}$ and Olav Reksen ${ }^{1}$ \\ ${ }^{1}$ Faculty of Veterinary Medicine, Department of Production Animal Clinical Sciences, Norwegian University of Life Sciences, PO Box 369 Sentrum, \\ N-0102 Oslo, Norway \\ ${ }^{2}$ TINE SA, PO Box 58, N-1430 Ås, Norway \\ ${ }^{3}$ GD Animal Health, Arnsbergstraat 7, 7400 AA Deventer, the Netherlands \\ ${ }^{4}$ Department of Animal Sciences, Wageningen University, 6708 PB Wageningen, the Netherlands \\ ${ }^{5}$ Department of Population Medicine and Diagnostic Sciences, Cornell University, Ithaca, NY 14850
}

\section{ABSTRACT}

Timely and accurate identification of cows with intramammary infections is essential for optimal udder health management. Various sensor systems have been developed to provide udder health information that can be used as a decision support tool for the farmer. Among these sensors, the DeLaval Online Cell Counter (DeLaval, Tumba, Sweden) provides somatic cell counts from every milking at cow level. Our aim was to describe and evaluate diagnostic sensor properties of these online cell counts (OCC) for detecting an intramammary infection, defined as an episode of subclinical mastitis or a new case of clinical mastitis. The predictive abilities of a single OCC value, rolling averages of OCC values, and an elevated mastitis risk (EMR) variable were compared for their accuracy in identifying cows with episodes of subclinical mastitis or new cases of clinical mastitis. Detection of subclinical mastitis episodes by OCC was performed in 2 separate groups of different mastitis pathogens, Pat 1 and Pat 2 , categorized by their known ability to increase somatic cell count. The data for this study were obtained in a field trial conducted in the dairy herd of the Norwegian University of Life Sciences. Altogether, 173 cows were sampled at least once during a 17 -mo study period. The total number of quarter milk cultures was 5,330. The most common Pat 1 pathogens were Staphylococcus epidermidis, Staphylococcus aureus, and Streptococcus dysgalactiae. The most common Pat 2 pathogens were Corynebacterium bovis, Staphylococcus chromogenes, and Staphylococcus haemolyticus. The OCC were successfully recorded from 82,182 of 96,542 milkings during

Received June 28, 2018.

Accepted February 15, 2019.

*Corresponding author: gunnar.dalen@nmbu.no the study period. For episodes of subclinical mastitis the rolling 7-d average OCC and the EMR approach performed better than a single OCC value for detection of Pat 1 subclinical mastitis episodes. The EMR approach outperformed the OCC approaches for detection of Pat 2 subclinical mastitis episodes. For the 2 pathogen groups, the sensitivity of detection of subclinical mastitis episodes was 69\% (Pat 1) and 31\% (Pat 2), respectively, at a predefined specificity of $80 \%$ (EMR). All 3 approaches were equally good at detecting new cases of clinical mastitis, with an optimum sensitivity of $80 \%$ and specificity of $90 \%$ (single OCC value).

Key words: intramammary infection, sensor, somatic cell count, online cell count

\section{INTRODUCTION}

From an economic perspective, mastitis is one of the most important diseases in dairy production (Halasa et al., 2007; Hogeveen et al., 2011). Much of the economic losses are due to reduced milk production following subclinical mastitis (Hogan et al., 2016). Therefore, detection and management of both subclinical and clinical mastitis are of importance for milk quality, animal welfare, and economic return.

The SCC can to some extent be used for the surveillance of IMI (Schukken et al., 2003), and the industry has advanced toward developing new sensors that are specifically designed for udder health surveillance. One of these is the DeLaval Online Cell Counter (DeLaval, Tumba, Sweden). With this, we can obtain repeated measurements of online cell counts (OCC) at cow level. These may be implemented in automated detection systems for the management of udder health in automatic milking systems (AMS).

Several studies of associations between SCC and IMI have used treatment of clinical mastitis as the gold standard for their evaluation (Kamphuis et al., 2008, 2013; Sørensen et al., 2016). However, these studies used different sensors to estimate SCC. Sørensen et 
al. (2016) used direct optical counting of somatic cells, whereas Kamphuis et al. $(2008,2013)$ used an indirect measurement of SCC based on viscosity measurements. Also, the ability of OCC to discriminate between IMI and physiological fluctuations in SCC not related to IMI has not been reported. This may be because longitudinal studies of IMI are both time consuming and costly, and because the detection of IMI is not straightforward.

An elevated SCC is usually a response to an IMI (IDF, 2013), and we are therefore measuring response to an infection, rather than the infection itself. Automated detection of the response to an IMI by using OCC must therefore take into account that the immune systems of different cows may respond differently to the same IMI pathogens (Rivas et al., 2013). Thus, the OCC from different cows with IMI due to the same pathogen may vary. However, with frequent sampling of OCC we may be able to distinguish between measurement noise and true changes resulting from biological processes. That is, arbitrary changes in OCC can be viewed as within-animal deviations and corrected for by calculating rolling averages or by using smoothing functions (Sørensen et al., 2016). To detect cases of clinical cases of mastitis, Sørensen et al. (2016) created an elevated mastitis risk (EMR) indicator, based on smoothed OCC. The EMR is a continuous variable (from $0-1$ ), where values close to 0 indicate low risk of mastitis and higher values, approaching 1 , indicate an increased risk of mastitis (Sørensen et al., 2016).

The OCC can also be elevated due to reasons other than IMI, including other systemic diseases, stage of lactation, stress, trauma, previous IMI, milking interval, day-to-day variation, and diurnal variation (IDF, 2013). Thus, the ability to distinguish between elevated OCC due to IMI or for other reasons is crucial for udder health management. Detection systems with a high specificity are often preferred by farmers using AMS (Claycomb et al., 2009) because a large number of false-positive alerts is a concern (Hogeveen et al., 2010). The diagnostic test properties of sensor systems should therefore be investigated and reported, so that farmers have an evidence-based foundation for choosing systems that match their requirements.

The primary aim of this study was to detect episodes of subclinical mastitis caused by mastitis pathogens. A secondary aim was to detect new cases of clinical mastitis. Specifically, we first wanted to test the predictive abilities of single values and rolling averages of OCC and an EMR indicator for detection of periods of subclinical mastitis or new cases of clinical mastitis. Second, we wanted to compare the diagnostic properties of these different approaches.

\section{MATERIALS AND METHODS}

\section{Field Study}

Data were obtained during a 17-mo longitudinal observational study in the research herd at the Norwegian University of Life Sciences. Two groups, each of approximately 50 Norwegian Red cows, housed in immediate proximity to each other, were milked on average 2.6 times per day by 2 identical AMS (DeLaval VMS) during the study period. The monthly mean number of lactating cows was 96, the mean milk production per cow per day was $27.9 \mathrm{~kg}$, and the average cow composite OCC was 115,103 cells $/ \mathrm{mL}$. The farm had reliable identification of animals and used standardized mastitis control practices, such as monthly milk quality testing in a DHIA program, postmilking teat disinfection, and selective dry-cow therapy.

The OCC were recorded from every milking from January 5, 2016, to May 22, 2017.

Trained veterinary personnel collected quarter milk samples (QMS) from all lactating cows on a monthly basis according to recommended guidelines (Hogan et al., 1999). Samples were frozen after collection and during transport to the laboratory for microbiological analyses. Samples were thawed in the laboratory and bacteriological culture was performed according to standard procedures (Hogan et al., 1999). Briefly, 0.1 $\mathrm{mL}$ of milk from each quarter was spread on cattle blood agar plates with esculin and incubated at $37^{\circ} \mathrm{C}$. Plates were read at 24 and $48 \mathrm{~h}$. Species identification of cultured bacteria was performed with MALDI-TOF MS microflex LT (Bruker Corporation, Billerica, MA; Cheuzeville, 2015). Samples with culture results indicating more than 2 morphologically different colony types were treated as contaminated and excluded from further analyses.

\section{Mastitis Status}

Based on the culture results, the cows were given an udder health status for subclinical mastitis throughout the study period. In this way, every milking was either associated with an episode of subclinical mastitis or not. A cow was considered to have an episode of subclinical mastitis when meeting at least 1 of the following criteria: (1) $\geq 1,000 \mathrm{cfu} / \mathrm{mL}$ of a single mastitis pathogen was cultured from a single sample in at least 1 quarter, (2) $\geq 500 \mathrm{cfu} / \mathrm{mL}$ of a mastitis pathogen was cultured from 2 out of 3 consecutive milk samples from the same quarter, or $(3) \geq 100 \mathrm{cfu} / \mathrm{mL}$ of a mastitis pathogen was cultured from 3 consecutive milk samples from the same quarter. These definitions were adapted 
from those of Zadoks et al. (2002). Cows with positive milk cultures that did not meet any of the above criteria were classified as being transiently colonized (Reksen et al., 2012).

Because the OCC is recorded at the cow level, the bacteriological diagnoses at the quarter level were aggregated into cow-level diagnoses. The same cow could experience an episode of subclinical mastitis in more than 1 quarter simultaneously, and in some cases, these 2 episodes could be caused by different mastitis pathogens. Hence, pathogens were divided into 2 groups (Pat $\mathbf{1}$ and Pat 2), according to characteristics of the bacteria. The group of pathogens from which a high cell count would be expected during a subclinical mastitis episode was named Pat 1 . The Pat 1 group consisted of the following species: Staphylococcus aureus, Streptococcus dysgalactiae, Streptococcus uberis, Enterococcus faecalis, Enterococcus faecium, Lactococcus lactis, Staphylococcus epidermidis, and Staphylococcus simulans (Djabri et al., 2002; Reksen et al., 2008; Simojoki et al., 2009, 2011; Fry et al., 2014). Known mastitis pathogens not included in Pat 1 were assigned to the Pat 2 category. These Pat 2 pathogens included Corynebacterium bovis, Staphylococcus chromogenes, Staphylococcus haemolyticus, Aerococcus viridans, Staphylococcus hominis, Staphylococcus xylosus, and other bacteria cultured.

Cows were given the status of subclinical mastitis when 1 or more quarters were positive for either a subclinical mastitis with a Pat 1 mastitis pathogen or a Pat 2 mastitis pathogen. For milkings where a cow was found positive for subclinical mastitis for mastitis pathogens from both categories (Pat 1 and Pat 2) simultaneously, we regarded the OCC response to be primarily due to the mastitis pathogen in the Pat 1 category. That is, we implemented a hierarchical order in the classification such that a cow could only be assigned to the Pat 2 subclinical mastitis category when there was no simultaneous diagnosis of a Pat 1 subclinical mastitis in the same cow during the same infectious period.

As sampling was performed monthly, we did not know exactly when each episode of subclinical mastitis started, and duration of infection was therefore calculated using the mid-point estimation method previously described by Zadoks et al. (2002). Thus, the start of the subclinical mastitis episode was defined as the middle of the time interval between a negative culture and the first positive culture event, and the end of the subclinical mastitis episode was defined as the middle of the time interval between the last positive culture event and the first negative culture for a cow defined as cured (Zadoks et al., 2002).

A veterinary treatment for clinical mastitis was defined as a new case of clinical mastitis.
Farm personnel identified cows with suspected clinical mastitis based on generalized clinical symptoms, including anorexia, lethargy, or elevated rectal temperature. These cows underwent clinical examination by the herd veterinarian. Cows that were treated for clinical mastitis were transferred to a treatment pen without AMS, and we do not have OCC records throughout the period of treatment for the clinical mastitis cases. Therefore, the last milking in the AMS before veterinary treatment was associated with each new case of clinical mastitis.

\section{OCC}

Statistical analyses were conducted using Stata (Stata SE/14, Stata Corp., College Station, TX).

The raw OCC values were smoothed using 1 of 3 different methods: (1) rolling 7-d average of available OCC, (2) rolling 48-h average of available OCC, and (3) calculation of the EMR for all milkings. The rolling 7-d and 48-h average of OCC were not transformed before calculating rolling averages and correspond to the values given in the AMS software DelPro (DeLaval). The EMR was computed as described by Sørensen et al. (2016) for all milkings. Briefly, we checked the validity of all recorded OCC measurements before lntransformation. Only milkings from 5 to 305 DIM with a milking interval of 4 to $24 \mathrm{~h}$ and a milking yield of $\geq 3.5 \mathrm{~kg}$ were included. Online cell count values of 0 were omitted from further analyses. We used the Wood lactation curve to calculate lactation-specific OCC curves for first, second, and third and above lactations (Wood, 1967). Periods with missing OCC data were corrected for by slowly approaching the lactation-specific OCC curves by $5 \%$ for each milking with missing observations (Sørensen et al., 2016). The ln-transformed OCC data were adjusted for aberrations and drift at the sensor level by single exponential smoothing (Hyndman et al., 2008) before double exponential smoothing of the adjusted OCC values was employed according to the description by Sørensen et al. (2016).

The lactation-specific OCC curves were used for rapid initialization of the double exponential smoothing (Sørensen et al., 2016). The output from the double exponential smoothing (level and trend) were used to calculate EMR values on a continuous scale from 0 to 1 (Sørensen et al., 2016).

\section{Diagnostic Test Properties}

The raw OCC values, rolling 7-d OCC values, and the EMR were evaluated against the subclinical mastitis status of the cow at every milking. For subclinical mastitis, the diagnostic properties were explored 
separately for the 2 pathogen groups, Pat 1 and Pat 2 . Furthermore, the raw OCC values, rolling 48-h OCC values and the EMR were evaluated against the clinical mastitis status of the cow at every milking. For each of the approaches, alert thresholds were calculated for 4 different levels of specificity for detection of Pat 1 subclinical mastitis episodes, Pat 2 subclinical mastitis episodes, and new cases of clinical mastitis using the "roctab" and "diagt" functions in Stata (Stata SE/14, Stata Corp.). The "roctab" function was used to identify the cut-point for each predefined level of specificity. It uses all registered outcomes of the diagnostic test variable as a classification cut-point and computes the corresponding sensitivity and specificity. The sensitivity of the identified diagnostic test variable cut-point for specificities of $80,85,90$, and $99 \%$ were further evaluated with "diagt." This displays summary statistics for a diagnostic test as compared with the true status, in our case an episode of subclinical mastitis or a new case of clinical mastitis. Alert thresholds for each level of specificity for Pat 2 subclinical mastitis were calculated after removing observations with a Pat 1 subclinical mastitis alert at the same level of specificity. The 4 different detection approaches were compared using the "roccomp" function in Stata, which compares the area under multiple receiver operating characteristics curves (Stata SE/14, Stata Corp.). The receiver operating characteristics area under the curve for detection of Pat 2 subclinical mastitis were calculated after removing observations with Pat 1 subclini- cal mastitis alerts at the level of $80 \%$ specificity for Pat 1 subclinical mastitis.

\section{RESULTS}

\section{Field Study}

Altogether, we collected 5,330 QMS from a total of 257 lactations in 173 cows. Each cow was on average sampled 8 times, ranging from 1 to 16 . Cows entered the study with an average of 38 DIM, ranging from 4 to 269. The average DIM of cows at sampling was 119 , ranging from 5 to 303 . Bacteria were cultured from 1,222 samples, with 1 and 2 pathogens cultured in 1,152 and 67 samples, respectively. We excluded 3 samples from the analysis due to contamination. Pathogens were found in 222 lactations in 155 cows. The distribution of bacterial culture results are shown in Table 1 .

\section{Mastitis Status}

According to our definition of subclinical mastitis status, there were 106 cow-level episodes of Pat 1 subclinical mastitis during the course of the study. These episodes were based on 324 positive cow-level culture results. A total of 23,409 AMS milkings from 97 lactations in 80 cows were associated with these episodes of Pat 1 subclinical mastitis. An additional 65 Pat 1 positive cow-level samples from 55 lactations in 53 cows did not meet any of our defined criteria for subclinical

Table 1. Distribution of 1,286 microbiological diagnoses in 1,219 samples with positive bacteriological culture results out of 5,327 quarter milk samples

\begin{tabular}{|c|c|c|c|c|}
\hline \multirow[b]{2}{*}{ Bacterial species detected } & \multirow[b]{2}{*}{$\mathrm{N}$} & \multicolumn{3}{|c|}{ Culture result $(\mathrm{cfu} / \mathrm{mL})$} \\
\hline & & $\geq 100$ and $<500$ & $\geq 500$ and $<1,000$ & $\geq 1,000$ \\
\hline Staphylococcus epidermidis & 234 & 54 & 36 & 144 \\
\hline Corynebacterium bovis & 225 & 31 & 41 & 153 \\
\hline Staphylococcus chromogenes & 167 & 14 & 12 & 141 \\
\hline Staphylococcus aureus & 119 & 45 & 11 & 63 \\
\hline Staphylococcus haemolyticus & 116 & 43 & 17 & 56 \\
\hline Aerococcus viridans & 91 & 57 & 14 & 20 \\
\hline $\begin{array}{l}\text { Enterococcus faecalis, Enterococcus faecium, } \\
\text { and Lactococcus lactis }\end{array}$ & 81 & 16 & 3 & 62 \\
\hline Streptococcus dysgalactiae & 66 & 9 & 3 & 54 \\
\hline Staphylococcus simulans & 32 & 6 & 5 & 21 \\
\hline Staphylococcus hominis & 31 & 19 & 6 & 6 \\
\hline Streptococcus uberis & 25 & 1 & 2 & 22 \\
\hline Staphylococcus xylosus & 8 & 1 & 1 & 6 \\
\hline Streptococcus agalactiae & - & - & - & - \\
\hline Other $^{1}$ & 91 & 48 & 17 & 26 \\
\hline Not detected & 4,108 & & & \\
\hline
\end{tabular}


Table 2. Pathogen-specific 7-d average cow-level online cell count (OCC) at the time of microbiological sampling in cows with subclinical mastitis

\begin{tabular}{|c|c|c|c|}
\hline Pathogens in episodes of subclinical mastitis & $\mathrm{N}$ & $\begin{array}{c}\text { OCC } \\
(1,000 \text { cells } / \mathrm{mL})\end{array}$ & $95 \% \mathrm{CI}$ \\
\hline Staphylococcus epidermidis & 141 & 191 & $150-232$ \\
\hline Corynebacterium bovis & 104 & 114 & $77-150$ \\
\hline Staphylococcus chromogenes & 136 & 154 & 111-197 \\
\hline Staphylococcus aureus & 77 & 355 & $281-430$ \\
\hline Staphylococcus haemolyticus & 67 & 112 & $82-142$ \\
\hline Aerococcus viridans & 20 & 119 & $14-224$ \\
\hline $\begin{array}{l}\text { Enterococcus faecalis, Enterococcus faecium, } \\
\text { and Lactococcus lactis }\end{array}$ & 56 & 292 & $183-400$ \\
\hline Streptococcus dysgalactiae & 56 & 308 & $204-412$ \\
\hline Staphylococcus simulans & 27 & 310 & $217-404$ \\
\hline Staphylococcus hominis & 6 & 119 & $0-309$ \\
\hline Streptococcus uberis & 22 & 298 & $162-434$ \\
\hline Staphylococcus xylosus & 7 & 180 & $122-238$ \\
\hline Other ${ }^{1}$ & 25 & 131 & $76-185$ \\
\hline
\end{tabular}

${ }^{1}$ Other bacteria cultured: Acinetobacter lwoffii, Bacillus pumilus, Corynebacterium amycolatum, Corynebacterium spp., Corynebacterium stationis, Macrococcus caseolyticus, Macrococcus luteus, Staphylococcus auricularis, Staphylococcus capitis, Staphylococcus equorum, Staphylococcus hyicus, Staphylococcus spp., Streptococcus canis, Streptococcus lutetiensis, Streptococcus spp., and Trueperella pyogenes.

mastitis and were classified as being transiently colonized.

Similarly, 117 episodes of Pat 2 subclinical mastitis occurred during the study period. These were associated with 288 positive cow-level culture results. A total of 22,182 milkings from 107 lactations in 84 cows were associated with these episodes of Pat 2 subclinical mastitis. An additional 106 Pat 2 positive cow-level culture results from 55 lactations in 51 cows were isolated in connection with an episode of Pat 1 subclinical mastitis. Following our hierarchical definition of subclinical mastitis, these culture results were not included in the Pat 2 subclinical mastitis episodes. Furthermore, 101 Pat 2 positive cow-level culture results from 78 lactations in 70 cows did not meet any of our defined criteria for subclinical mastitis and were classified as being transiently colonized.

During the study period, we recorded 16 veterinary treatments for clinical mastitis in 15 cows.
OCC

The OCC was successfully recorded from 82,182 of 96,542 milkings; the 14,360 missing values were because of equipment failure or failure to service and refill the OCC unit with reagent.

The Pat 1 subclinical mastitis pathogens were generally associated with a higher OCC than the Pat 2 subclinical mastitis pathogens. An overview of the pathogen-specific subclinical mastitis diagnoses and corresponding 7 -d average OCC at the time of sampling are given in Table 2. The 7-d average OCC of cows with different subclinical mastitis status, as well as the 48-h average OCC of cows with a new case of clinical mastitis, are shown in Table 3. The 7-d average OCC of cows with Pat 1 subclinical mastitis and the 48-h average OCC of cows with a new case of clinical mastitis are clearly higher than the corresponding values from other groups, but this is not the case for Pat 2 subclinical

Table 3. Pathogen group-specific 7-d average of cow-level online cell count (OCC) values at the time of microbiological sampling in cows with subclinical mastitis (SCM), and average of cow-level OCC from milkings during the $48 \mathrm{~h}$ before a new case of clinical mastitis

\begin{tabular}{lccc}
\hline Group & \multicolumn{3}{c}{ OCC } \\
\hline Pat $^{1}$ 1 SCM & $\mathrm{N}$ & $(1,000$ cells $/ \mathrm{mL})$ & $95 \%$ CI \\
Pat 2 SCM & 311 & 260 & $224-298$ \\
Any SCM & 269 & 83 & $70-96$ \\
Transient colonization & 580 & 178 & $157-200$ \\
No SCM and no bacteria cultured from any teat in cow & 134 & 124 & $56-192$ \\
New case of clinical mastitis & 519 & 53 & $44-61$ \\
\hline${ }^{1}$ Pat & 16 & 1,280 & $721-1,838$ \\
\hline
\end{tabular}

${ }^{1}$ Pat $=$ pathogen group. 

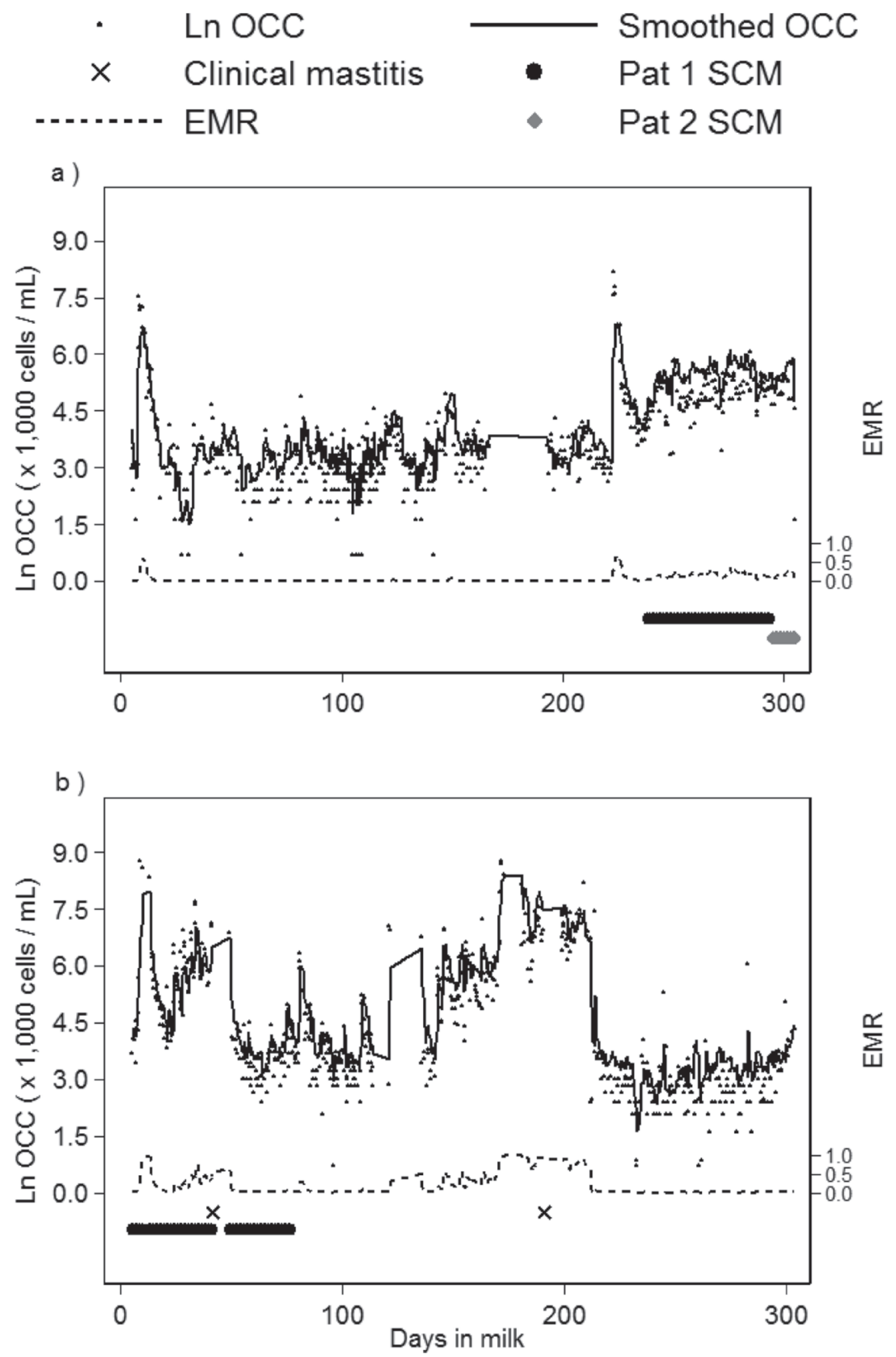

Figure 1. Examples of online cell count (OCC) values and mastitis status. The cow in (a) is a typical example of a cow with low OCC values until the onset of a case of Pat 1 subclinical mastitis (SCM). The Pat 1 SCM is followed by a case of Pat 2 SCM. This cow was not treated for clinical mastitis. The cow in (b) is an example of a cow with Pat 1 SCM and 2 treatments for clinical mastitis. EMR = elevated mastitis risk. Pat 1 and Pat $2=$ different groups of mastitis pathogens. 
Table 4. Sensitivities at set specificities for detection of cases of Pat 1 subclinical mastitis (SCM), cases of Pat 2 SCM, and new cases of clinical mastitis (CM) using online cell count (OCC) or elevated mastitis risk (EMR) values ${ }^{1}$

\begin{tabular}{|c|c|c|c|c|}
\hline Item & $\begin{array}{c}\mathrm{Sp}=80 \\
\text { Se }(95 \% \mathrm{CI})[\text { cut-off }]\end{array}$ & $\begin{array}{c}\mathrm{Sp}=85 \\
\text { Se }(95 \% \mathrm{CI})[\text { cut-off }]\end{array}$ & $\begin{array}{c}\mathrm{Sp}=90 \\
\text { Se }(95 \% \mathrm{CI})[\text { cut-off }]\end{array}$ & $\begin{array}{c}\mathrm{Sp}=99 \\
\text { Se }(95 \% \mathrm{CI}) \text { [cut-off }]\end{array}$ \\
\hline \multicolumn{5}{|l|}{ Pat 1 SCM } \\
\hline EMR & $69(68-69)[\geq 0.03]$ & $59(59-60)[\geq 0.05]$ & $48(48-49)[\geq 0.08]$ & $8(7-8)[\geq 0.62]$ \\
\hline \multicolumn{5}{|l|}{ Pat 2 SCM } \\
\hline Single OCC & $29(28-30)[\geq 40]$ & $20(19-20)[\geq 57]$ & $12(12-13)[\geq 88]$ & $0.5(0.4-0.7)[\geq 642]$ \\
\hline \multicolumn{5}{|l|}{ New CM } \\
\hline Single OCC & $87(60-98)[\geq 121]$ & $87(60-98)[\geq 159]$ & $80(52-96)[\geq 232]$ & $60(32-84)[\geq 1,397]$ \\
\hline 48-h average $\mathrm{OCC}$ & $81(54-96)[\geq 126]$ & $69(41-89)[\geq 167]$ & $69(41-89)[\geq 243]$ & $44(20-70)[\geq 1,336]$ \\
\hline EMR & $81(54-96)[\geq 0.08]$ & $81(54-96)[\geq 0.12]$ & $75(48-93)[\geq 0.20]$ & $38(15-65)[\geq 0.83]$ \\
\hline
\end{tabular}

${ }^{1}$ Cut-off values for the levels of sensitivity (Se) and specificity (Sp) are in 1,000 cells/mL for OCC and between 0 and 1 for the EMR. Calculated sensitivities for Pat 2 subclinical mastitis were conducted for each level of specificity after the removal of milkings with a sensor alert for subclinical Pat 1 subclinical mastitis at that level of specificity. Pat 1 and Pat $2=$ different groups of mastitis pathogens.

mastitis and transient colonizations. Examples of OCC curves and mastitis status are shown in Figure 1.

\section{Diagnostic Test Properties}

The diagnostic test properties for the predefined specificities of a single OCC value, the rolling 7 -d or 48-h average of OCC and the EMR, are shown distributed by Pat 1 subclinical mastitis episodes, Pat 2 subclinical mastitis episodes, and new cases of clinical mastitis in Table 4. The sensitivity of the different approaches decreases when higher specificity is demanded.

The receiver operating characteristics area under the curve of Pat 1 subclinical mastitis episodes, Pat 2 subclinical mastitis episodes, and new cases of clinical mastitis are shown in Table 5. In our study, we found that the EMR and the rolling 7-d average of OCC performed better than the single OCC for detection of Pat 1 subclinical mastitis episodes. The ROC curves of the different approaches for detecting Pat 1 subclinical mastitis episodes are shown in Figure 2. For the detection of Pat 2 subclinical mastitis episodes, the EMR approach performed better than both OCC approaches. The 3 different detection approaches performed equally well for detection of new cases of clinical mastitis. The ROC curves of the different approaches for detecting new cases of clinical mastitis are shown in Figure 2.

\section{DISCUSSION}

This is a study exploring the practical application of OCC to detect episodes of subclinical mastitis and new cases of clinical mastitis. The study is limited to one farm, which hampers the generalizability of the results. However, the aim of the study was to test the practical applicability of the approach rather than to generalize the results on a larger proportion of the population.

In this study we demonstrate that OCC may be used to identify cows with an episode of subclinical mastitis and new cases of clinical mastitis. The diagnostic test properties of the system can be adapted according to the required practical application, with settings selected on the basis of the tradeoff between sensitivity and specificity. A farmer with a high tolerance of false positives may choose to increase the sensitivity at the cost of lower specificity.

No systems, including our approach in this study, currently operate at the desired level for sensor systems

Table 5. Receiver operating characteristics area under the curve (ROC area) of the 3 detection approaches for detection of cases of Pat 1 subclinical mastitis (SCM), Pat 2 SCM, and new cases of clinical mastitis $(\mathrm{CM})^{1}$

\begin{tabular}{lcc}
\hline Detection approach & $\begin{array}{c}\text { ROC } \\
\text { area }\end{array}$ & $95 \%$ CI \\
\hline Pat 1 SCM & & \\
$\quad$ Single OCC & & \\
Rolling 7-d average OCC & 0.783 & $0.779-0.787$ \\
EMR $^{3}$ & 0.809 & $0.806-0.813$ \\
Pat 2 SCM & 0.804 & $0.800-0.808$ \\
$\quad$ Single OCC & 0.587 & $0.581-0.593$ \\
Rolling 7-d average OCC & 0.597 & $0.591-0.603$ \\
EMR & 0.641 & $0.635-0.647$ \\
New CM & & \\
Single OCC & 0.931 & $0.859-1.000$ \\
Rolling average 48 h OCC & 0.925 & $0.869-0.980$ \\
EMR & 0.904 & $0.802-1.000$ \\
\hline
\end{tabular}

${ }^{1}$ The ROC area under the curve for detection of Pat 2 subclinical mastitis is calculated after excluding milkings with Pat 1 subclinical mastitis alerts at the level of $80 \%$ specificity for Pat 1 subclinical mastitis. Pat 1 and Pat $2=$ different groups of mastitis pathogens.

${ }^{2} \mathrm{OCC}=$ online cell count.

${ }^{3} \mathrm{EMR}=$ elevated mastitis risk. 
Rolling average OCC
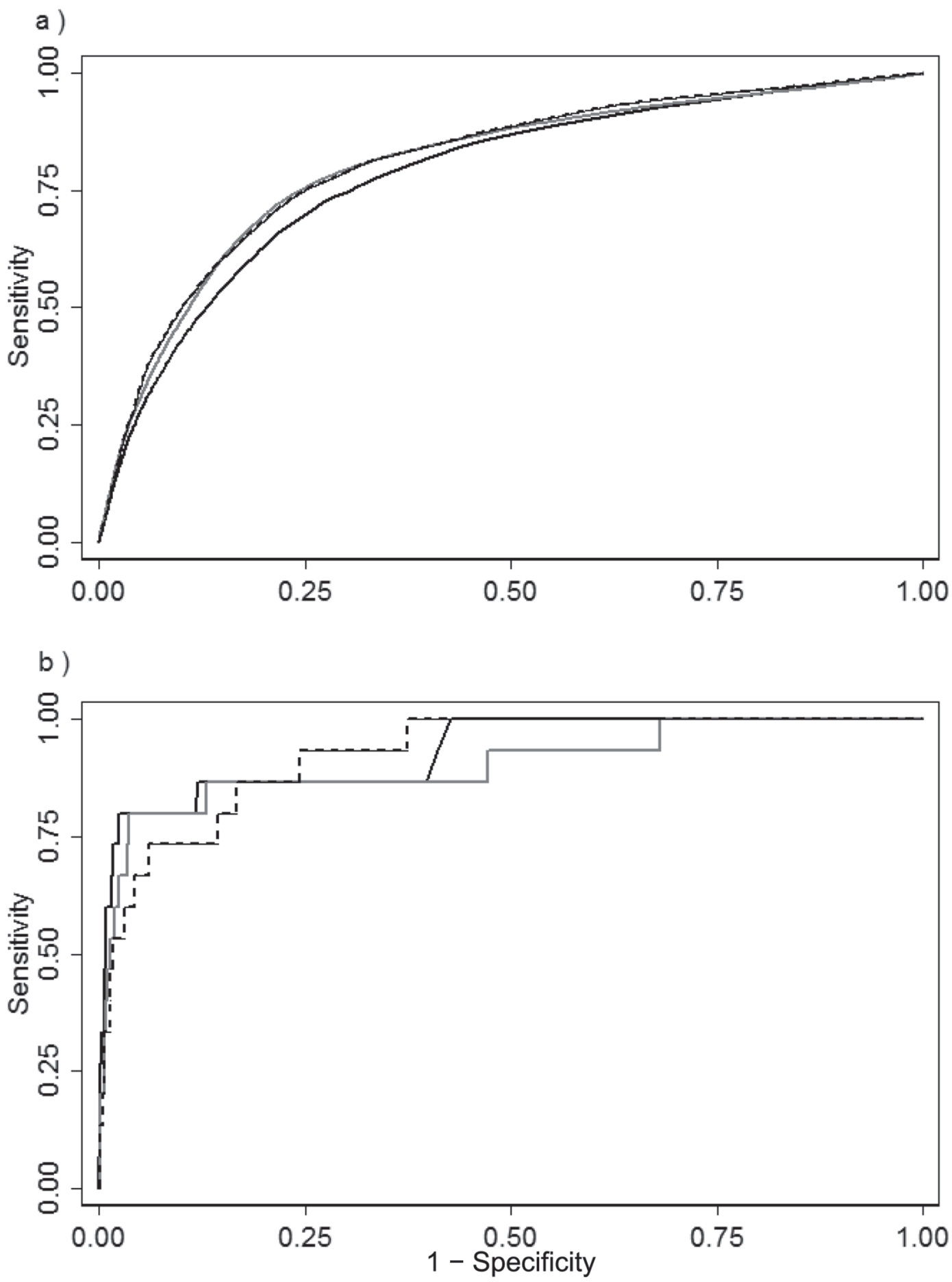

Figure 2. Receiver operating characteristic curves for detection of (a) Pat 1 subclinical mastitis and (b) new cases of clinical mastitis. The time windows for rolling averages are $7 \mathrm{~d}$ and $48 \mathrm{~h}$ for (a) and (b), respectively. EMR = elevated mastitis risk; OCC = online cell count. Pat 1 and Pat 2 = different groups of mastitis pathogens. 
in dairy production (ISO, 2007). We suggest that using sensor systems could still be advantageous for the herd manager and that the usefulness of the system depends on the desired application. Thus, we suggest that sensitivity should be high for detection of cows for dry-cow treatment, but specificity should be high for detection of IMI during lactation. This suggestion is related to the number of detection events. For selective dry-cow therapy, for which there is a single detection event, high sensitivity at the cost of lower specificity will result in a moderate number of false-positive IMI alerts. However, a test with similar characteristics will lead to numerous false alerts during lactation, when every sensor measurement would represent a possible detection event. Both single OCC values and smoothed values, like the EMR of Sørensen et al. (2016), can be used to detect episodes of subclinical mastitis and new cases of clinical mastitis. Based on this, we propose that systems should be adjusted according to the lactation stage of the individual cow and the tolerance of the farmer for false positives, such that farm-specific everyday practical udder health management is accommodated. This is in line with current work undertaken by the International Dairy Federation (Hogeveen et al., 2018).

Sørensen et al. (2016) used treatment of mastitis with antibiotics as their gold standard for mastitis cases, but they also suggested that the EMR could identify cows with chronic infections. From our data, the single EMR and the rolling 7-d average OCC performed better than a single OCC value for the detection of Pat 1 subclinical mastitis episodes. Furthermore, the EMR approach performed better than both the OCC approaches for the detection of Pat 2 subclinical mastitis episodes. We suggest that this reflects the variation in immune response between individuals (Rivas et al., 2013), and that the cow-specific smoothing inherent in the EMR provides more information that improves the diagnostic properties of the system.

Our results shows that the confidence interval (CI) of the 7-d average OCC for episodes of Pat 1 subclinical mastitis or new cases of clinical mastitis differed from those of the other groups. The corresponding CI for Pat 2 subclinical mastitis episodes and transient colonization overlapped, and the CI of transient colonization overlapped with those of cows with both no subclinical mastitis and no transient colonization. This overlap in CI makes it difficult to separate the latter groups from each other. However, as the aim is to manage udder health, as measured by bulk tank SCC, the most important goal is to identify cows with a true IMI accompanied by a high SCC.
We consider the results are applicable to dairy cows with frequent OCC measurements in AMS. However, Sørensen et al. (2016) reported large differences in test algorithm performance between herds. Assuming that this is the case for our study also, a limitation of the external validity of our results is that we have only studied a single herd that may have a quite specific OCC pattern. Furthermore, because the results are based on OCC only, they cannot be extrapolated to other udder health sensors or SCC from DHI samples without further evaluation.

Our aim was to use OCC measurements to detect cows with subclinical mastitis associated with infection over time or high colony-forming unit counts of mastitis pathogens. We chose bacteriological culture of QMS combined with our criteria of infection over time or high colony-forming unit counts to define episodes of subclinical mastitis. This is an imperfect gold standard and the results may be biased by misclassification of subclinical mastitis status (Dohoo et al., 2011). Because of our conservative definition of subclinical mastitis status, there were likely few false positive cases. Therefore, the misclassification mainly includes cows with subclinical mastitis that were falsely defined as healthy. This results in a negative bias in specificity. Furthermore, as cows were sampled for bacteriological culture once monthly, some cows may have had an episode of subclinical mastitis between our visits. When cured, these would not be detected by bacteriological culture, but they might have been identified by the sensor system. Also, the sensor system may have detected the true start and end of subclinical mastitis episodes, whereas our defined start and end were set by the midpoint estimation method described. Thus, we may have treated the sensor alerts as false, although they could actually have been correct. This is a challenge for all detection approaches that are based on SCC (IDF, 2013).

We grouped the bacteriological diagnoses in our study in the Pat 1 and Pat 2 groups, and not the traditional major and minor pathogen groups. This was because the non-aureus staphylococci is a heterogeneous group of bacterial species (Vanderhaeghen et al., 2015). In our study, we included Staphylococcus epidermidis and Staphylococcus simulans of the non-aureus staphylococci in the group expected to cause elevated OCC (Pat 1). This was based on reports of these pathogens' ability to cause IMI over time and elevated SCC (Simojoki et al., 2011; Fry et al., 2014). Furthermore, we chose a hierarchical approach to grouping bacteriological diagnoses in Pat 1 subclinical mastitis episodes and Pat 2 subclinical mastitis episodes, such that a cow could 
not be positive for both Pat 1 subclinical mastitis and Pat 2 subclinical mastitis simultaneously. The reason for choosing this approach was that Pat 1 subclinical mastitis episodes were likely to have a greater effect on the OCC than Pat 2 subclinical mastitis episodes. The benefit of this approach is that we could evaluate the ability of the system to identify episodes of Pat 2 subclinical mastitis after removing cows with Pat 1 subclinical mastitis alerts. Thus, the system divided the herd into 4 mastitis status groups: cows with Pat 1 subclinical mastitis episodes, cows with Pat 2 subclinical mastitis episodes, cows with no subclinical mastitis and cows with a new case of clinical mastitis.

Rapid detection of clinical mastitis is important for both animal welfare, milk quality, and economic return. At the same time, more data may improve the diagnostic test properties of sensor equipment (Hogeveen et al., 2010). To balance this issue, the rolling average OCC for detection of clinical mastitis in our study was set to use OCC data from $48 \mathrm{~h}$. This did, however, not improve the detection of new cases of clinical mastitis in our study. Although, in our study, all 3 approaches performed equally for the detection of new cases of clinical mastitis, we have relatively few cases. There could be differences in the operating characteristics of the 3 approaches for the detection of new cases of clinical mastitis that we are unable to estimate with our material. Also, because we do not have OCC readings throughout the clinical mastitis treatment period, we cannot evaluate which approach is best for continued alerts for clinical mastitis. However, the sensitivities and specificities are both likely to improve when the time window for matching the gold standard of clinical mastitis is increased (Hogeveen et al., 2010).

Furthermore, to improve the detection of subclinical mastitis in our study, the rolling average OCC for detection of subclinical mastitis was set to use data from $7 \mathrm{~d}$. This significantly improved the diagnostic test properties for detection of Pat 1 subclinical mastitis episodes. We propose that this longer detection window is acceptable because subclinical mastitis does not necessarily require immediate action for animal welfare reasons.

Frequent sensor alerts can be a concern for herd managers. Therefore, information from the sensor system has to be actionable, and the level of alert should be adapted to the urgency of the situation. That is, whenever there is an alert, the system should be able to evaluate whether there is a need for immediate notification of the herd manager. Furthermore, optimal actions should be suggested, with predictions of outcome for each suggestion. For the convenience of the user and to limit overtreatment of cows, the number of false positives should be minimized.

\section{CONCLUSIONS}

We investigated detection of subclinical mastitis episodes and new cases of clinical mastitis based on OCC from every milking. For diagnosis of Pat 1 subclinical mastitis episodes, the EMR and a rolling 7-d average of OCC outperformed a single OCC value. For diagnosis of Pat 2 subclinical mastitis episodes, the EMR outperformed the OCC approaches. For detection of new cases of clinical mastitis, all approaches performed equally well. By combining different alerts, the systems can be adapted to the needs of individual farmers regarding udder health management in their herds.

\section{ACKNOWLEDGMENTS}

The authors acknowledge the contribution of participating farm staff. The support of our field and laboratory staff is also greatly appreciated. The data for this study were available from the project "New approaches to management and breeding, in automatic milking systems." The financial sources were TINE SA, DeLaval, Geno SA (Hamar, Norway), the Norwegian Research Funding for Agriculture and the Food Industry (Oslo, Norway), and the Norwegian Research Council (Lysaker, Norway).

\section{REFERENCES}

Cheuzeville, L. 2015. Rapid detection of microorganisms in the dairy value chain by MALDI-TOF MS. MS Thesis. Faculty of Science and Technology, University of Stavanger, Stavanger, Norway.

Claycomb, R. W., P. T. Johnstone, G. A. Mein, and R. A. Sherlock. 2009. An automated in-line clinical mastitis detection system using measurement of conductivity from foremilk of individual udder quarters. N. Z. Vet. J. 57:208-214.

Djabri, B., N. Bareille, F. Beaudeau, and H. Seegers. 2002. Quarter milk somatic cell count in infected dairy cows: A meta-analysis. Vet. Res. 33:335-357.

Dohoo, I., S. Andersen, R. Dingwell, K. Hand, D. Kelton, K. Leslie, Y. Schukken, and S. Godden. 2011. Diagnosing intramammary infections: Comparison of multiple versus single quarter milk samples for the identification of intramammary infections in lactating dairy cows. J. Dairy Sci. 94:5515-5522.

Fry, P. R., J. R. Middleton, S. Dufour, J. Perry, D. Scholl, and I. Dohoo. 2014. Association of coagulase-negative staphylococcal species, mammary quarter milk somatic cell count, and persistence of intramammary infection in dairy cattle. J. Dairy Sci. 97:4876-4885.

Halasa, T., K. Huijps, O. Osteras, and H. Hogeveen. 2007. Economic effects of bovine mastitis and mastitis management: A review. Vet. Q. 29:18-31.

Hogan, J. S., E. A. Berry, J. E. Hillerton, H. Hogeveen, S. C. Nickerson, S. P. Oliver, G. M. Pighetti, P. Rapnicki, Y. H. Schukken, and K. L. Smith. 2016. Current concepts of bovine mastitis. 5th ed. Current concepts of bovine mastitis. National Mastitis Council, Madison, WI.

Hogan, J. S., R. N. González, S. C. Harmon, S. C. Nickerson, J. W. Pankey, and K. L. Smith. 1999. Laboratory handbook on bovine mastitis. 1st ed. National Mastitis Council, Madison, WI. 
Hogeveen, H., G. Dalen, and I. C. Klaas. 2018. Rethinking definitions for mastitis detection: Leaving the paradigm of only clinical mastitis. IDF Animal Health Report 12:23-24.

Hogeveen, H., K. Huijps, and T. J. Lam. 2011. Economic aspects of mastitis: New developments. N. Z. Vet. J. 59:16-23.

Hogeveen, H., C. Kamphuis, W. Steeneveld, and H. Mollenhorst. 2010. Sensors and clinical mastitis-The quest for the perfect alert. Sensors (Basel) 10:7991-8009.

Hyndman, R., A. Koehler, K. Ord, and R. Snyder. 2008. Forecasting with Exponential Smoothing: The State Space Approach. The State Space Approach. Springer Berlin Heidelberg, Berlin, Germany.

IDF. 2013. Guidelines for the use and interpretation of bovine somatic cell counts (SCC) in the dairy industry. Bull. Int. Dairy Fed. Int. Dairy Fed., Brussels, Belgium.

ISO. 2007. ISO 20966:2007. Automatic milking systems - Requirements and testing. Annex C: Example of methods of evaluating detection systems for milk demand as abnormal due to blood or changes in homogeneity. International Organization for Standardization, Geneva, Switzerland.

Kamphuis, C., B. Dela Rue, G. Mein, and J. Jago. 2013. Development of protocols to evaluate in-line mastitis-detection systems. J. Dairy Sci. 96:4047-4058

Kamphuis, C., R. Sherlock, J. Jago, G. Mein, and H. Hogeveen. 2008. Automatic detection of clinical mastitis is improved by in-line monitoring of somatic cell count. J. Dairy Sci. 91:4560-4570.

Reksen, O., Y. T. Gröhn, J. W. Barlow, and Y. H. Schukken. 2012 Transmission dynamics of intramammary infections with coagulase-negative staphylococci. J. Dairy Sci. 95:4899-4910.

Reksen, O., L. Solverod, and O. Osteras. 2008. Relationships between milk culture results and composite milk somatic cell counts in Norwegian dairy cattle. J. Dairy Sci. 91:3102-3113.
Rivas, A. L., M. D. Jankowski, R. Piccinini, G. Leitner, D. Schwarz, K. L. Anderson, J. M. Fair, A. L. Hoogesteijn, W. Wolter, M. Chaffer, S. Blum, T. Were, S. N. Konah, P. Kempaiah, J. M. Ong'echa, U. S. Diesterbeck, R. Pilla, C. P. Czerny, J. B. Hittner, J. M. Hyman, and D. J. Perkins. 2013. Feedback-based, system-level properties of vertebrate-microbial interactions. PLoS One 8:e53984.

Schukken, Y. H., D. J. Wilson, F. Welcome, L. Garrison-Tikofsky, and R. N. Gonzalez. 2003. Monitoring udder health and milk quality using somatic cell counts. Vet. Res. 34:579-596.

Simojoki, H., T. Orro, S. Taponen, and S. Pyorala. 2009. Host response in bovine mastitis experimentally induced with Staphylococcus chromogenes. Vet. Microbiol. 134:95-99.

Simojoki, H., T. Salomaki, S. Taponen, A. Iivanainen, and S. Pyorala. 2011. Innate immune response in experimentally induced bovine intramammary infection with Staphylococcus simulans and S. epidermidis. Vet. Res. (Faisalabad) 42:49.

Sørensen, L. P., M. Bjerring, and P. Lovendahl. 2016. Monitoring individual cow udder health in automated milking systems using online somatic cell counts. J. Dairy Sci. 99:608-620.

Vanderhaeghen, W.. S. Piepers, F. Leroy, E. Van Coillie, F. Haesebrouck, and S. De Vliegher. 2015. Identification, typing, ecology and epidemiology of coagulase negative staphylococci associated with ruminants. Vet. J. 203:44-51.

Wood, P. D. P. 1967. Algebraic model of lactation curve in cattle. Nature 216:164-165.

Zadoks, R. N., H. G. Allore, T. J. Hagenaars, H. W. Barkema, and Y. H. Schukken. 2002. A mathematical model of Staphylococcus aureus control in dairy herds. Epidemiol. Infect. 129:397-416. 